\title{
First-passage and escape problems in the Feller process
}

\author{
Jaume Masoliver* and Josep Perelló ${ }^{\dagger}$ \\ Departament de Física Fonamental, Universitat de Barcelona, Diagonal, 647, E-08028 Barcelona, Spain
}

(Received 13 September 2012; published 10 October 2012; corrected 12 October 2012)

\begin{abstract}
The Feller process is an one-dimensional diffusion process with linear drift and state-dependent diffusion coefficient vanishing at the origin. The process is positive definite and it is this property along with its linear character that have made Feller process a convenient candidate for the modeling of a number of phenomena ranging from single-neuron firing to volatility of financial assets. While general properties of the process have long been well known, less known are properties related to level crossing such as the first-passage and the escape problems. In this work we thoroughly address these questions.
\end{abstract}

DOI: 10.1103/PhysRevE.86.041116

\section{INTRODUCTION}

Diffusion processes are Markovian random processes with continuous sample paths. From a mathematical point of view they are characterized, in one dimension, by two functions: the drift, $f(y, t)$, and a positive defined diffusion coefficient $D(y, t) \geqslant 0$. The sample paths of any diffusion process can thus be pictured as the continuous trajectory resulting from the superposition of a deterministic evolution, governed by $f(y, t)$, and fluctuations around it, the latter determined by $D(y, t)$. Denoting the process by $Y(t)$, the diffusion picture becomes apparent by the fact that the time evolution of $Y(t)$ is ruled by the stochastic differential equation

$$
d Y(t)=f(Y(t), t) d t+\sqrt{D(Y(t), t)} d W(t),
$$

where $W(t)$ is the Wiener process, that is, a Gaussian process with zero mean, unit variance, and correlation function $\left\langle W\left(t_{1}\right) W\left(t_{2}\right)\right\rangle=\min \left(t_{1}, t_{2}\right)$. In what follows all stochastic differentials are interpreted in the sense of Ito.

The Feller process is a special kind of diffusion process with linear drift and linear diffusion coefficient vanishing at the origin [1]. The time evolution of the process is thus governed by

$$
d Y(t)=[-\alpha Y(t)+\beta] d t+k \sqrt{Y(t)} d W(t),
$$

where $\alpha>0, \beta$ and $k>0$ are constant parameters.

Both Feller and Ornstein-Uhlenbeck processes (diffusion processes also with linear drift but constant diffusion coefficient) have been widely used, with a marked prominence of the latter, in the modeling of countless physical phenomena. Both share a linear drift, $f(Y)=-\alpha Y+\beta$, which for $\alpha>0$ results in a restoring force that, in the absence of noise, makes both processes decay toward the value $\beta$.

However, and contrary to the Ornstein-Uhlenbek process where diffusion is constant, the Feller process has a statedependent diffusion coefficient, $D(Y)=k^{2} Y$, which for large values of $Y$ enhances the effects of noise while as $Y$ goes to zero the effect of noise vanishes. Hence, when the process reaches the origin, the drift drags it toward the value $\beta$. If $\beta>0$ the process, starting at some positive value, cannot reach the negative region which, in turn, renders the process always nonnegative [otherwise the noise term in Eq. (1) would become

\footnotetext{
*jaume.masoliver@ub.edu

†josep.perello@ub.edu
}

PACS number(s): 05.40.Jc, 89.65.Gh, 02.50.Ey, 05.45.Tp

imaginary]. Therefore, for the Feller process the origin is a singular boundary that the process cannot cross.

A related question is whether or not the origin is accessible, in other words, whether the value $Y=0$ can or cannot be attained by the process. This is a crucial question in many practical situations and, as we will prove later, the answer depends on the particular value taken by a parameter which balances the values of $\beta$ and $k^{2}$. The problem of classifying the different types of boundaries appearing in diffusion processes was thoroughly studied by Feller himself during the 1950s and we refer the reader to the literature for a more complete account on the subject [2-4].

Possessing linear drift and state-dependent diffusion and, most importantly, the fact that the process never attains negative values have made Feller process an ideal candidate for modeling a number of phenomena in natural and social sciences. Theoretical biology was one of the first places where the process was, during 1970s, seriously considered [5]. Perhaps the most prominent place is within the context of neurobiology in order to model the firing of single neurons [6-9]. The Feller neuronal model is one of the so-called stochastic integrateand-fire models which are simple representations aiming to reproduce the membrane potential fluctuations. Experimental progress has led to the possibility of fitting real data to the Feller neuronal model among others models [10-14].

In a different context Capocelli and Ricciardi [15] considered the possibility of representing biological populations with the Feller process in order to include environmental randomness to the classic Malthusian growth rate [16]. The approach $[15,17,18]$ represents in fact an alternative to the Lotka-Volterra models in ecosystems and the interest in this sort of problem is mostly focused on extinction (that is, on the possibility of attaining the singular boundary $Y=0$ ) as well as on unrestricted growth $[17,18]$.

Financial markets is another field where the Feller process is widely used. It was introduced in 1985 to represent the term structure of interest rates-receiving the name of the Cox, Ingersoll, and Ross (CIR) model—and it successfully evaluated bond prices $[19,20]$. The process is also being considered to provide a random character to the volatility of a given stock. Volatility obeying the Feller model jointly with a log-Brownian stochastic dynamics for the asset price evolution configure a two-dimensional diffusion market process called the Heston model [21,22] which is a rather useful model especially for option pricing $[20,21]$. 
In all of the above mentioned situations susceptible to being represented by a Feller process, the first-passage time events related, among others, to level crossing and the triggering of a given signal are very significant phenomena for a number of reasons which depend on each context. This is, for instance, the case of neuronal activity where spike generation is due to the crossing of a threshold by the membrane potential signal. Additionally, any sort of population extinction or, in a completely different context, the volatility bursts in financial markets are also important phenomena to model and study. First-passage times constitute, however, a difficult topic [23-27]. To our knowledge, in the case of the Feller process this crucial facet has been scantily studied and only partially solved some years ago in the context of single-neuron firing $[6,8]$. It is our main objective to address the first-passage time properties of process (1).

This paper is organized as follows. In Sec. II, we introduce the general properties of the unrestricted probability density of the Feller model. Section III is devoted to the derivation of the first-passage time and escape probabilities with special attention to a couple of specific situations. Section IV is mostly focused on the derivation of the mean first-passage time. We finally summarize the results obtained in Sec. V.

\section{GENERAL PROPERTIES OF THE FELLER MODEL}

Before addressing the main issue of this paper, let us briefly review the main traits of the process and the role of the boundary at the origin. For the rest of the paper in turns out to be convenient to scale time and the process itself in the following way (recall we have assumed $\alpha>0$ ):

$$
t^{\prime}=\alpha t, \quad X=\frac{2 \alpha}{k^{2}} Y,
$$

so that the Langevin equation (1) reads

$$
d X\left(t^{\prime}\right)=-\left[X\left(t^{\prime}\right)-\theta\right] d t^{\prime}+\sqrt{2 X\left(t^{\prime}\right)} d W\left(t^{\prime}\right),
$$

where $\theta$ is the only free parameter left. Its relation to $\beta$ and $k$ is

$$
\theta=\frac{2 \beta}{k^{2}}>0
$$

This parameter is called "saturation level" or "normal level" and it is the value to which $X\left(t^{\prime}\right)$ is attracted. As we will shortly see, $\theta$ has a key role in the behavior of the Feller process.

Let $p\left(x, t^{\prime} \mid x_{0}\right)$ be the probability density function (pdf) for process (3) to be in state $x$ at time $t^{\prime}$ :

$$
p\left(x, t^{\prime} \mid x_{0}\right) d x=\operatorname{Prob}\left\{x \leqslant X\left(t^{\prime}\right)<x+d x \mid X(0)=x_{0}\right\} .
$$

This density satisfies the (forward) Fokker-Planck equation (FPE) (as long as there is no confusion we will drop the prime in the time variable)

$$
\frac{\partial p}{\partial t}=\frac{\partial}{\partial x}[(x-\theta) p]+\frac{\partial^{2}}{\partial x^{2}}(x p),
$$

with initial condition

$$
p\left(x, 0 \mid x_{0}\right)=\delta\left(x-x_{0}\right) .
$$

Recall that $x=0$ is a singular boundary of the process and no "particle" can either leave or enter through this boundary (see Sec. I). A sufficient condition for this to happen is that the probability flux of the process through $x=0$ is zero [4]. We will thus search for solutions of the initial-value problem (5) and (6) that meet such a condition, that is,

$$
\lim _{x \rightarrow 0}\left\{(x-\theta) p\left(x, t \mid x_{0}\right)+\frac{\partial}{\partial x}\left[x p\left(x, t \mid x_{0}\right)\right]\right\}=0 .
$$

The expression for the pdf of the process $p\left(x, t \mid x_{0}\right)$ was first obtained by Feller himself many years ago using a tortuous procedure which involved the solution of a rather clumsy integral equation [1]. In Appendix A we present a simpler and more direct derivation based on the Laplace transform of the problem (5) and (6). The final expression reads

$$
\begin{aligned}
p\left(x, t \mid x_{0}\right)= & \frac{1}{1-e^{-t}}\left(\frac{x e^{-t}}{x_{0}}\right)^{(\theta-1) / 2} \\
& \times \exp \left\{-\frac{x+x_{0} e^{-t}}{1-e^{-t}}\right\} I_{\theta-1}\left(\frac{2 \sqrt{x x_{0} e^{-t}}}{1-e^{-t}}\right),
\end{aligned}
$$

where $I_{\theta-1}(z)$ is a modified Bessel function defined as [28]

$$
I_{\theta-1}(z)=\sum_{n=0}^{\infty} \frac{(z / 2)^{2 n+\theta-1}}{n ! \Gamma(n+\theta)} .
$$

From Eq. (8) we easily get the stationary pdf of the process defined as

$$
p_{\mathrm{st}}(x)=\lim _{t \rightarrow \infty} p\left(x, t \mid x_{0}\right) .
$$

Indeed, taking into account that [see Eq. (9)]

$$
I_{\theta-1}(z)=\frac{1}{\Gamma(\theta)}(z / 2)^{\theta-1}\left[1+O\left(z^{2}\right)\right],
$$

from Eq. (8) we directly obtain the Gamma distribution:

$$
p_{\mathrm{st}}(x)=\frac{1}{\Gamma(\theta)} x^{\theta-1} e^{-x} .
$$

Another property that we can easily establish is the behavior of the probability distribution at the singular boundary located at $x=0$. In effect, using Eq. (10) we see from Eq. (8) that

$$
p\left(x, t \mid x_{0}\right)=\frac{e^{-x_{0} e^{-t} /\left(1-e^{-t}\right)}}{\Gamma(\theta)\left(1-e^{-t}\right)^{\theta}} x^{\theta-1}[1+O(x)],
$$

from which it follows

$$
p\left(0, t \mid x_{0}\right)= \begin{cases}\infty, & \theta<1 \\ 0, & \theta>1\end{cases}
$$

and

$$
p\left(0, t \mid x_{0}\right)=\frac{e^{-x_{0} e^{-t} /\left(1-e^{-t}\right)}}{1-e^{-t}} \quad(\theta=1) .
$$

We thus see that when $\theta>1$ the probability for the Feller process to reach the value $x=0$ is zero but when $\theta \leqslant 1$ this probability is greater than zero. In other words, if $\theta \leqslant 1$ the origin is an accessible boundary, while if $\theta>1$ it is not [1,2].

\section{FIRST-PASSAGE AND ESCAPE PROBABILITIES}

After reviewing the main traits of the Feller process we will now focus on level-crossing problems-a collective name embracing questions such as hitting, first-passage, escape, and extreme values, among others-for that process. According to whether we are dealing with one-sided or two-sided barrier 
problems, we separate level crossing into two different issues. In one of them, the hitting or first-passage problem, we deal with the time that the process reaches some "critical" value, or "threshold," for the first time. The second issue, albeit closely related to the first one, concerns the time when the process first leaves a given interval. This is the so-called escape or exit problem.

\section{A. The first-passage probability}

Let us first address the first-passage problem for the Feller process. The problem is solved when one knows the firstpassage probability to threshold $x_{c}$. Let us denote by $W_{c}(t \mid x)$ the probability of first reaching $x_{c} \geqslant 0$ when the process starts at $t=0$ from the value $x>0$.

As is well known $[4,23,27]$ the first-passage probability satisfies the backward Fokker-Planck, or Kolmogorov, equation

$$
\frac{\partial W_{c}}{\partial t}=-(x-\theta) \frac{\partial W_{c}}{\partial x}+x \frac{\partial^{2} W_{c}}{\partial x^{2}} .
$$

Note that at $t=0$ the process cannot have reached the threshold as long as $x \neq x_{c}$. The following initial condition thus holds:

$$
W_{c}(0 \mid x)=0 .
$$

Moreover, if the initial value happens to be the threshold itself, $x=x_{c}$, then crossing will be a sure event at any time. We have, therefore, the following boundary condition:

$$
W_{c}\left(t \mid x_{c}\right)=1 \text {. }
$$

The difficulty of solving the initial-boundary problem (14)-(16) is decreased by taking the time Laplace transform,

$$
\hat{W}_{c}(s \mid x)=\int_{0}^{\infty} e^{-s t} W_{c}(t \mid x) d t,
$$

which reduces the original problem to the solution of an ordinary differential equation (the Kummer equation [28]):

$$
x \frac{d^{2} \hat{W}_{c}}{d x^{2}}-(x-\theta) \frac{d \hat{W}_{c}}{d x}-s \hat{W}_{c}=0,
$$

with boundary condition

$$
\hat{W}_{c}\left(s \mid x_{c}\right)=\frac{1}{s} .
$$

Since $W_{c}$ is a probability it is obvious that any solution of the problem must be finite and nonnegative for all $x \geqslant 0$.

The general solution of the Kummer equation (17) is [28]

$$
\hat{W}_{c}(s \mid x)=A F(s, \theta, x)+B U(s, \theta, x),
$$

where $A$ and $B$ are arbitrary constants and $F(s, \theta, x)$ and $U(s, \theta, x)$ are the confluent confluent hypergeometric functions of the first and second kind [28], respectively, defined by

$$
F(s, \theta, x)=\frac{\Gamma(\theta)}{\Gamma(s)} \sum_{n=0}^{\infty} \frac{\Gamma(s+n)}{\Gamma(\theta+n)} \frac{x^{n}}{n !}
$$

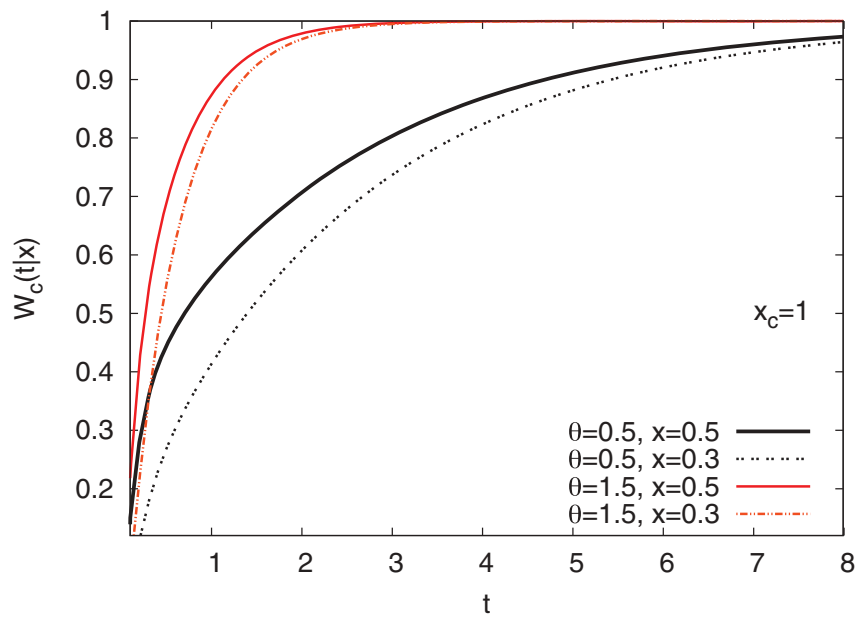

FIG. 1. (Color online) First-passage probability $W_{c}(t \mid x)$ given by Eq. (23) as a function of the scaled time and for two different parameters $\theta$ and initial values $x$. Inverse Laplace transform is obtained with the Stehfest algorithm.

and

$$
\begin{aligned}
U(s, \theta, x)= & \frac{\Gamma(1-\theta)}{\Gamma(s+1-\theta)} F(s, \theta, x) \\
& +\frac{\Gamma(\theta-1)}{\Gamma(s)} x^{\theta-1} F(s+1-\theta, 2-\theta, x) .
\end{aligned}
$$

In order to proceed further we need to specify whether the initial value $x$ is above or below the threshold $x_{c}$.

\section{Initial value below threshold $\left(x \leqslant x_{c}\right)$}

In this case $x$ can be arbitrarily small and taking into account that [see Eqs. (20) and (21)]

$$
\lim _{x \rightarrow 0} U(s, \theta, x)= \begin{cases}\frac{\Gamma(1-\theta)}{\Gamma(s+1-\theta)}, & \theta<1, \\ \infty, & \theta>1,\end{cases}
$$

we see that the solution to the problem staying finite for any initial position between the origin and $x_{c}$ and for any positive value of the parameter $\theta$ is

$$
\hat{W}_{c}(s \mid x)=A F(s, \theta, x) .
$$

The boundary condition (18) fixes the value of $A$ and

$$
\hat{W}_{c}(s \mid x)=\frac{F(s, \theta, x)}{s F\left(s, \theta, x_{c}\right)} \quad\left(x \leqslant x_{c}\right) .
$$

Figure 1 shows the numerical computation of this expression in the original Laplace domain. We have used the wellknown Stehfest algorithm [29] and the results do not show any computational problem. As expected the closest to the threshold the fastest the first-passage time probability decays. And a greater $\theta$ corresponds to a smaller $W_{c}(t \mid x)$, as well.

\section{Initial value above threshold $\left(x \geqslant x_{c}\right)$}

In such a case $x$ can be arbitrarily large. Hence, taking into account that

$$
\lim _{x \rightarrow \infty} F(s, \theta, x)=\infty,
$$

while $U(s, \theta, x)$ stays finite for all positive values of $x$ [28], we see from Eq. (19) that the general solution of the problem 
which remains finite for all $x>0$ is

$$
\hat{W}_{c}(s \mid x)=B U(s, \theta, x),
$$

and from the boundary condition (18) we conclude

$$
\hat{W}_{c}(s \mid x)=\frac{U(s, \theta, x)}{s U\left(s, \theta, x_{c}\right)} \quad\left(x \geqslant x_{c}\right) .
$$

Numerical inversion of this result is again easy to compute with standard algorithms. The small difference lies in the fact that the confluent hypergeometric function of the second kind (21) is slightly more complicated than the confluent hypergeometric function of the first kind (20).

\section{B. Reaching the origin}

Another interesting quantity is the first-passage probability to threshold $x_{c}=0$, that is to say, the probability of first attaining the singular boundary of the process. This probability is relevant in the firing of neurons and it was addressed some years ago by Capocelli and Ricciardi [6] (see also the work of Laska et al. [8]). Let us denote by $W_{0}$ the first-passage probability to the origin. Since our process is always positive, $x \geqslant 0$, we must use Eq. (24) in order to evaluate $\hat{W}_{0}$. Setting $x_{c}=0$ in Eq. (24) and using Eq. (22) we obtain

$$
\hat{W}_{0}(s \mid x)= \begin{cases}\frac{\Gamma(s+1-\theta)}{s \Gamma(1-\theta)} U(s, \theta, x), & \theta<1, \\ 0, & \theta>1 .\end{cases}
$$

We will now proceed to invert Eq. (25) thus obtaining the first-passage probability $W(t \mid x)$ in real time, something that seems to be unfeasible for any value of the threshold $x_{c}$, at least exactly (more on this below).

Using the property [28]

$$
U(s, \theta, x)=x^{1-\theta} U(s+1-\theta, 2-\theta, x),
$$

we write for $\theta<1$

$$
\hat{W}_{0}(s \mid x)=\frac{1}{s} \frac{\Gamma(s+1-\theta)}{\Gamma(1-\theta)} x^{1-\theta} U(s+1-\theta, 2-\theta, x),
$$

which, after using the following integral representation of the Kummer function $U$ [28],

$$
U(a, c, x)=\frac{1}{\Gamma(a)} \int_{0}^{\infty} e^{-x z} z^{a-1}(1+z)^{c-a-1} d z
$$

reads

$$
\hat{W}_{0}(s \mid x)=\frac{x^{1-\theta}}{s \Gamma(1-\theta)} \int_{0}^{\infty} e^{-x z} z^{-\theta}\left(\frac{z}{1+z}\right)^{s} d z .
$$

Therefore,

$$
W_{0}(t \mid x)=\frac{x^{1-\theta}}{\Gamma(1-\theta)} \int_{0}^{\infty} e^{-x z} z^{-\theta} \mathcal{L}^{-1}\left\{\frac{1}{s}\left(\frac{z}{1+z}\right)^{s}\right\} d z,
$$

where $\mathcal{L}^{-1}$ stands for Laplace inversion. Since [30]

$$
\mathcal{L}^{-1}\left\{\frac{e^{-a s}}{s}\right\}=\Theta(t-a)
$$

where $\Theta(\cdot)$ is the Heaviside step function, we have

$$
\begin{aligned}
\mathcal{L}^{-1}\left\{\frac{1}{s}\left(\frac{z}{1+z}\right)^{s}\right\} & =\Theta\left[t+\ln \left(\frac{z}{1+z}\right)\right] \\
& =\Theta\left(z-\frac{e^{-t}}{1-e^{-t}}\right) .
\end{aligned}
$$

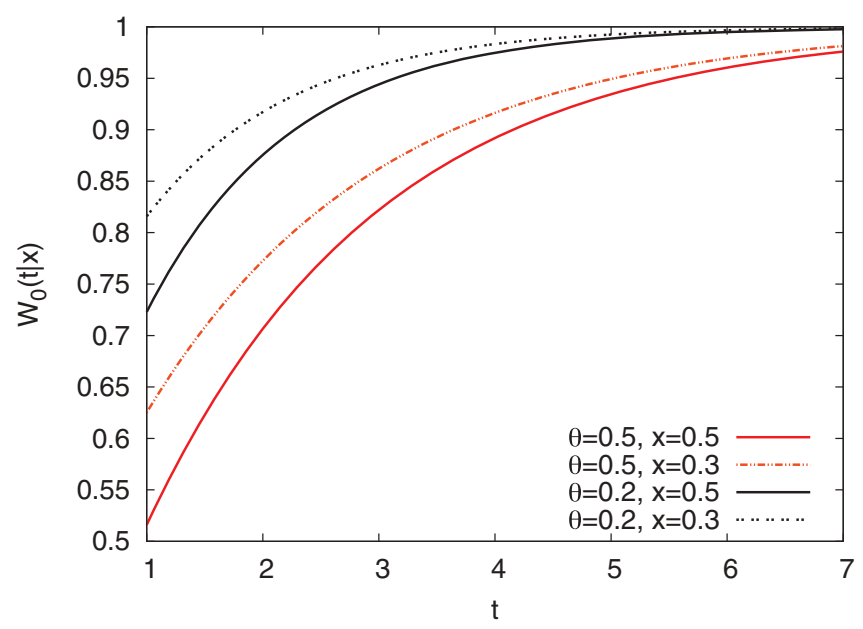

FIG. 2. (Color online) First-passage probability $W_{0}(t \mid x)$ given by Eq. (27) as a function of time $(t>1)$ for two different values of $\theta$ $(\theta<1)$ and the initial values $x$. Note that smaller values of $\theta$ and $x$ imply bigger first-passage probability.

Hence,

$$
W_{0}(t \mid x)=\frac{x^{1-\theta}}{\Gamma(1-\theta)} \int_{e^{-t} /\left(1-e^{-t}\right)}^{\infty} e^{-x z} z^{-\theta} d z,
$$

or, equivalently,

$$
W_{0}(t \mid x)=\frac{1}{\Gamma(1-\theta)} \Gamma\left(1-\theta, \frac{x e^{-t}}{1-e^{-t}}\right),
$$

where $\Gamma(a, z)$ is the incomplete Gamma function [28]

$$
\Gamma(a, z)=\int_{z}^{\infty} y^{a-1} e^{-y} d y .
$$

Finally,

$$
W_{0}(t \mid x)= \begin{cases}\frac{1}{\Gamma(1-\theta)} \Gamma\left(1-\theta, \frac{x e^{-t}}{1-e^{-t}}\right), & \theta<1, \\ 0, & \theta>1 .\end{cases}
$$

We remark [as shown already in Eq. (25)] that when $\theta>1$ the first-passage probability to the origin is zero in agreement with the fact, pointed out in Sec. II, that if $\theta>1 x=0$ is unattainable. The probability of first reaching the origin is represented in Fig. 2 where $W_{0}(t \mid x)$ is shown as a function of time and for two different values of parameter model $\theta$ and initial value $x$.

As $t \rightarrow \infty$ and for $\theta<1$ the first-passage probability $W_{0}$ becomes equal to 1 , as is expected since if $\theta<1$ crossing the origin is a certain event as time grows. It is, however, interesting to see how $W_{0}$ approaches unity. To this end we use the following series expansion of the incomplete Gamma function [28]:

$$
\Gamma(a, z)=\Gamma(a)-\sum_{n=0}^{\infty} \frac{(-1)^{n}}{n !} \frac{z^{a+n}}{a+n} .
$$

In the limit $t \rightarrow \infty$ we then get

$$
\begin{aligned}
& \Gamma\left(1-\theta, \frac{x e^{-t}}{1-e^{-t}}\right) \\
& \quad=\Gamma(1-\theta)-\frac{1}{1-\theta} x^{1-\theta} e^{-(1-\theta) t}+O\left(e^{-(2-\theta) t}\right) .
\end{aligned}
$$


Therefore,

$$
\begin{aligned}
W_{0}(t \mid x)= & 1-\frac{1}{\Gamma(2-\theta)} x^{1-\theta} e^{-(1-\theta) t} \\
& +O\left(e^{-(2-\theta) t}\right) \quad(\theta<1)
\end{aligned}
$$

an interesting expression combining a power law in $x$ and an exponential decay in time.

\section{Large threshold}

We will now study in some detail the interesting case of a large value of the threshold which is the opposite case considered above. It is clear that as $x_{c} \rightarrow \infty$ the threshold becomes unreachable and the first-passage probability approaches zero. Let us see how the limiting process is. Let us incidentally mention that these results are of real interest in econophysics when one, for instance, wants to control financial asset volatilities since large volatilities correspond to wild fluctuations in asset prices.

In the case under consideration the initial position is always below threshold and the starting point of the analysis must be Eq. (23)

$$
\hat{W}_{c}(s \mid x)=\frac{F(s, \theta, x)}{s F\left(s, \theta, x_{c}\right)} .
$$

Since now $x_{c} \rightarrow \infty$ we use the following asymptotic expansion of the Kummer function $F$ [28]

$$
F\left(s, \theta, x_{c}\right)=\frac{\Gamma(\theta)}{\Gamma(s)} e^{x_{c}} x_{c}^{s-\theta}\left[1+O\left(\frac{1}{x_{c}}\right)\right]
$$

and

$$
\hat{W}_{c}(s \mid x)=\frac{\Gamma(s)}{s \Gamma(\theta)} e^{-x_{c}} x_{c}^{\theta-s} F(s, \theta, x)\left[1+O\left(\frac{1}{x_{c}}\right)\right] .
$$

Our next step is the use of the following integral representation of $F[28]$ :

$$
F(s, \theta, x)=\frac{1}{\Gamma(s)} \int_{0}^{\infty} e^{-z} z^{s-1} F(\theta, x z) d z,
$$

where $F(\theta, x z)$ is the following hypergeometric series [28],

$$
F(\theta, x z)=\sum_{n=0}^{\infty} \frac{1}{(\theta)_{n}} \frac{(x z)^{n}}{n !} .
$$

Hence, for large values of the threshold, we approximately have

$$
\hat{W}_{c}(s \mid x) \simeq \frac{1}{s \Gamma(\theta)} e^{-x_{c}} x_{c}^{\theta-s} \int_{0}^{\infty} e^{-z} z^{s-1} F(\theta, x z) d z\left(x_{c} \rightarrow \infty\right) .
$$

We now proceed as in Sec. III B. The Laplace inversion of the last equation reads

$$
\begin{aligned}
W_{c}(t \mid x) \simeq & \frac{1}{\Gamma(\theta)} e^{-x_{c}} x_{c}^{\theta} \int_{0}^{\infty} \frac{e^{-z}}{z} F(\theta, x z) \\
& \mathcal{L}^{-1}\left\{\frac{1}{s}\left(\frac{z}{x_{c}}\right)^{s}\right\} d z \quad\left(x_{c} \rightarrow \infty\right) .
\end{aligned}
$$

Since

$$
\left(\frac{z}{x_{c}}\right)^{s}=\exp \left[s \ln \left(\frac{z}{x_{c}}\right)\right]
$$

then, recalling Eq. (26), we have

$$
\mathcal{L}^{-1}\left\{\frac{1}{s}\left(\frac{z}{x_{c}}\right)^{s}\right\}=\Theta\left[t+\ln \left(\frac{z}{x_{c}}\right)\right]=\Theta\left(z-x_{c} e^{-t}\right) .
$$

Therefore,

$$
W_{c}(t \mid x) \simeq \frac{1}{\Gamma(\theta)} x_{c}^{\theta} e^{-x_{c}} \int_{x_{c} e^{-t}}^{\infty} \frac{e^{-z}}{z} F(\theta, x z) d z \quad\left(x_{c} \rightarrow \infty\right) .
$$

Using Eq. (29) we can give an alternative expression to Eq. (30) which is somewhat more convenient for numerical work. It reads

$W_{c}(t \mid x) \simeq \frac{1}{\Gamma(\theta)} x_{c}^{\theta} e^{-x_{c}} \sum_{n=0}^{\infty} \frac{1}{(\theta)_{n}} \frac{x^{n}}{n !} \Gamma\left(n, x_{c} e^{-t}\right) \quad\left(x_{c} \rightarrow \infty\right)$,

where $\Gamma\left(n, x_{c} e^{-t}\right)$ is the incomplete Gamma function. This expression is particularly suited for small values of the initial position. Thus, for instance, when $x=0$ we write

$$
W_{c}(t \mid 0) \simeq \frac{1}{\Gamma(\theta)} x_{c}^{\theta} e^{-x_{c}} E_{1}\left(x_{c} e^{-t}\right) \quad\left(x_{c} \rightarrow \infty\right),
$$

where

$$
E_{1}(x)=\int_{x}^{\infty} \frac{e^{-z}}{z} d z
$$

is the exponential integral.

\section{The escape probability}

We close this section by briefly addressing the escape problem which, as mentioned before, is closely related with the first-passage problem studied above. The problem at hand consists in knowing whether or not the process $X(t)$, starting at some point inside an interval $(a, b)$, has left this interval for the first time. The answer lies in the knowledge of the survival probability $S_{a b}(t \mid x)$ defined as the probability that, starting at $x \in(a, b)$, the process at time $t$ has not left the interval at that time or during any previous instant of time:

$$
S_{a b}(t \mid x)=\operatorname{Prob}\left\{a<X\left(t^{\prime}\right)<b ; 0 \leqslant t^{\prime} \leqslant t \mid a<x<b\right\},
$$

where $x=X(0)$ is the starting point. The escape probability, i.e., the probability that at time $t$ the process has exited the interval $(a, b)$ for the first time, is then given by

$$
W_{a b}(t \mid x)=1-S_{a b}(t \mid x) .
$$

As is well known [4,23,27] the survival probability obeys the backward Fokker-Planck equation

$$
\frac{\partial S_{a b}}{\partial t}=-(x-\theta) \frac{\partial S_{a b}}{\partial x}+x \frac{\partial^{2} S_{a b}}{\partial x^{2}},
$$

with initial and boundary conditions given by

$$
S_{a b}(0 \mid x)=1, \quad S_{a b}(t \mid a)=S_{a b}(t \mid b)=0 .
$$

Hence, the escape probability is the solution of the initial and boundary value problem [compare with 
Eqs. (14)-(16)]

$$
\begin{gathered}
\frac{\partial W_{a b}}{\partial t}=-(x-\theta) \frac{\partial W_{a b}}{\partial x}+x \frac{\partial^{2} W_{a b}}{\partial x^{2}}, \\
W_{a b}(0 \mid x)=0, \quad W_{a b}(t \mid a)=W_{a b}(t \mid b)=1 .
\end{gathered}
$$

Following the same reasoning as before [see Eqs. (17) and (18)] we see that the time Laplace transform of the escape probability $\hat{W}(s \mid x)$ satisfies the boundary value problem

$$
x \frac{d^{2} \hat{W}_{a b}}{d x^{2}}-(x-\theta) \frac{d \hat{W}_{a b}}{d x}-s \hat{W}_{a b}=0,
$$

$$
\hat{W}_{a b}(s \mid a)=\hat{W}_{a b}(s \mid b)=\frac{1}{s} .
$$

Again, the general solution of the Kummer equation (35) is [28]

$$
\hat{W}_{a b}(s \mid x)=A F(s, \theta, x)+B U(s, \theta, x),
$$

where $A$ and $B$ are arbitrary constants and $F$ and $U$ are defined in Eqs. (20) and (21).

Boundary conditions (36) determine the value of $A$ and $B$ and after routine algebra the final result for the escape probability reads

$$
\begin{aligned}
\hat{W}_{a b}(s \mid x) & =\frac{[U(s, \theta, b)-U(s, \theta, a)] F(s, \theta, x)-[F(s, \theta, b)-F(s, \theta, a)] U(s, \theta, x)}{s[F(s, \theta, a) U(s, \theta, b)-F(s, \theta, b) U(s, \theta, a)]} \\
a & \leqslant x \leqslant b .
\end{aligned}
$$

\section{LONG-TIME ASYMPTOTIC BEHAVIOR AND MEAN FIRST-PASSAGE TIMES}

In the previous section we have solved the hitting and escape problems for the Feller process by means of the evaluation of the first-passage and exit probabilities. We have obtained exact analytical expressions for the time-Laplace transform of these probabilities. Unfortunately exact inversion seems to be beyond reach except for the cases in Sec. III B-when the threshold is located at the origin-and in the following Sec. IV A-with approximate expressions suitable for long times. In this section we will also obtain two important magnitudes associated with the problem: the mean firstpassage time, $T_{c}(x)$, and the mean escape time, $T_{a b}(x)$, which, in turn, allow for the long-time asymptotic expressions of the hitting and escape probabilities as we will first see next.

\section{A. Long-time behavior of the first-passage probability}

Let $\tau_{c}(x)$ be the first-passage time for the process, starting at $x$, to reach some threshold $x_{c}$ for the first time. It is a random variable depending on each realization of the process. Formally

$$
\tau_{c}(x)=\inf \left\{t \mid X(t)>x_{c} ; X(0)=x<x_{c}\right\}
$$

when the initial value is below threshold, and

$$
\tau_{c}(x)=\inf \left\{t \mid X(t)<x_{c} ; X(0)=x>x_{c}\right\}
$$

when the initial value is above threshold.

We next relate the first-passage time with the hitting probability $W_{c}(t \mid x)$ defined in the previous section. Note that if $\tau_{c}(x)$ is the first-passage time, the hitting probability can be defined as

$$
W_{c}(t \mid x)=\operatorname{Prob}\left\{\tau_{c}(x) \leqslant t\right\},
$$

which shows that $W_{c}(t \mid x)$ is the distribution function of the first-passage time. The corresponding probability density is thus defined

$$
f_{c}(t \mid x) d t=\operatorname{Prob}\left\{t \leqslant \tau_{c}(x)<t+d t\right\},
$$

and it is related to the distribution $W_{c}$ by

$$
f_{c}(t \mid x)=\frac{\partial W_{c}(t \mid x)}{\partial t} .
$$

The moments of this distribution are

$$
T_{n}\left(x \mid x_{c}\right)=\int_{0}^{\infty} t^{n} f_{c}(t \mid x) d t
$$

( $n=1,2,3, \ldots)$, and the mean first-passage time (MFPT) is the first moment:

$$
T_{c}(x) \equiv T_{1}\left(x \mid x_{c}\right)
$$

Note that in terms of the Laplace transform

$$
\hat{f}_{c}(s \mid x)=\int_{0}^{\infty} e^{-s t} f_{c}(t \mid x) d t,
$$

the first-passage moments are

$$
T_{n}\left(x \mid x_{c}\right)=\left.(-1)^{n} \frac{\partial^{n} \hat{f}_{c}(s \mid x)}{\partial s^{n}}\right|_{s=0},
$$

which implies that, as long as $T_{n}\left(x \mid x_{c}\right)(n=1,2,3, \ldots)$ exist, the Laplace transform of the first-passage time density has the following expansion in powers of $s$ :

$$
\hat{f_{c}}(s \mid x)=\sum_{n=0}^{\infty} \frac{(-1)^{n}}{n !} T_{n}\left(x \mid x_{c}\right) s^{n} .
$$

On the other hand the Laplace transform of Eq. (38), along with the initial condition $W_{c}(0 \mid x)=0$, yields

$$
\hat{W}_{c}(s \mid x)=\frac{1}{s} \hat{f}(s \mid x) .
$$

By combining Eqs. (39) and (40) we then have

$$
\hat{W}_{c}(s \mid x)=\sum_{n=0}^{\infty} \frac{(-1)^{n}}{n !} s^{n-1} T_{n}\left(x \mid x_{c}\right),
$$

expansion that furnishes the basis for the asymptotic analysis of the first-passage probability $W(t \mid x)$. Indeed, the so-called 
Tauberian theorems prove that the long-time behavior of a function $g(t)$ is determined by the small $s$ behavior of its Laplace transform $\hat{g}(s)$ [31]. For the case of the first-passage probability we see from Eq. (41) that the small $s$ behavior of $\hat{W}_{c}$ is

$$
\hat{W}_{c}(s \mid x)=\frac{1}{s}-T_{c}(x)+O(s)=\frac{1}{s}\left[1-s T_{c}(x)+O\left(s^{2}\right)\right],
$$

where $T_{c}(x) \equiv T_{1}\left(x \mid x_{c}\right)$ is the mean first-passage time. Note that expansion (42) may also be written, within the same level of approximation, as

$$
\hat{W}_{c}(s \mid x)=\frac{1}{s\left[1+s T_{c}(x)+O\left(s^{2}\right)\right]},
$$

which by the Tauberian theorems [31] implies that the longtime behavior of the first-passage probability $W_{c}(t \mid x)$ is given by the Laplace inversion of Eq. (43). That is,

$$
W_{c}(t \mid x) \simeq 1-e^{-t / T_{c}(x)} \quad(t \rightarrow \infty) .
$$

We have thus obtained the long-time behavior of the first-passage probability to threshold $x_{c}$ and see that the MFPT determines the long-time behavior of the firstpassage probability. We will next evaluate this average time for the Feller process.

\section{B. The mean first-passage time}

In terms of the Laplace transform of first-passage probability $\hat{W}_{c}(s \mid x)$ obtaining the MFPT is straightforward. In effect from Eq. (42) we see that

$$
T_{c}(x)=\lim _{s \rightarrow 0}\left[\frac{1}{s}-\hat{W}_{c}(s \mid x)\right] .
$$

Using the findings of Sec. III we know that the first-passage probability has different expressions as to whether the initial value of the process $x$ is above or below the threshold $x_{c}$. Let us now treat these two cases including the special case $x_{c}=0$.

\section{Initial value above threshold $\left(x \geqslant x_{c}\right)$}

In this case [see Eq. (24)]

$$
\hat{W}_{c}(s \mid x)=\frac{U(s, \theta, x)}{s U\left(s, \theta, x_{c}\right)}
$$

and

$$
T_{c}(x)=\lim _{s \rightarrow 0}\left[\frac{1}{s} \frac{U\left(s, \theta, x_{c}\right)-U(s, \theta, x)}{U\left(s, \theta, x_{c}\right)}\right] .
$$

The expansion in powers of $s$ of the Kummer function $U(s, \theta, x)$ is presented in Appendix A where it is shown that

$$
U(s, \theta, x)=1+s U_{1}(x)+O\left(s^{2}\right),
$$

where

$$
U_{1}(x) \equiv-\psi(1-\theta)-\int^{x} U(1,1+\theta, z) d z,
$$

and $\psi(z)=\Gamma^{\prime}(z) / \Gamma(z)$ is the psi function. We note that the function, defined as the indefinite integral

$$
\int^{x} U(1,1+\theta, z) d z
$$

cannot be reduced to another Kummer function [28] or, to the best of our knowledge, to any other tabulated function.

Plugging Eqs. (47) and (48) into Eq. (46) we finally obtain

$$
T_{c}(x)=\int_{x_{c}}^{x} U(1,1+\theta, z) d z \quad\left(x \geqslant x_{c}\right) .
$$

\section{MFPT to the origin}

In Secs. II and III [see Eq. (25)] we have seen that when $\theta>$ 1 the origin is unattainable. However, if $\theta<1$ this singular boundary can be reached by the process. In this later case it is natural to ask which is the MFPT to the origin. The question has not only an academic interest but is relevant in mathematical biology where $x=0$ corresponds to the potential at which a neuron is fired [6]. Also in econophysics it is useful to know whether volatility or the interest rates can drop to zero and what is the average time expected to do so.

Since $x>0$, the expression for the MFPT to the origin, denoted by $T_{0}(x)$, will be given by Eq. (49) with $x_{c}=0$. Unfortunately setting $x_{c}=0$ in Eq. (49) is not possible because the integral is singular at the lower level.

We proceed as follows: Start with the definition of the Kummer function $U$ given in Eq. (21), use the integration rule [28]

$$
\int^{x} F(a, c, z) d z=\frac{x^{c}}{c} F(a, c+1, x),
$$

and take into account the standard property of the Gamma function $\Gamma(z+1)=z \Gamma(z)$. We write

$$
\begin{aligned}
\int^{x} U(1,1+\theta, z) d z= & -\frac{1}{\theta} \int^{x} F(1,1+\theta, z) d z \\
& -\Gamma(\theta-1) x^{1-\theta} F(1-\theta, 2-\theta, x) .
\end{aligned}
$$

Substituting into Eq. (49) and taking the limit $x_{c} \rightarrow 0^{+}$, we have

$$
\begin{aligned}
T_{0}(x)= & \lim _{x_{c} \rightarrow 0^{+}}\left\{-\frac{1}{\theta} \int_{x_{c}}^{x} F(1,1+\theta, z) d z-\Gamma(\theta-1)\left[x^{1-\theta}\right.\right. \\
& \left.\left.\times F(1-\theta, 2-\theta, x)-x_{c}^{1-\theta} F\left(1-\theta, 2-\theta, x_{c}\right)\right]\right\} .
\end{aligned}
$$

Using the value of the Kummer function $F$ at the origin, $F(1,1+\theta, 0)=1$ [see Eq. (20)], we have

$$
\begin{aligned}
T_{0}(x)= & -\frac{1}{\theta} \int_{0}^{x} F(1,1+\theta, z) d z-\Gamma(\theta-1) \\
& \times\left[x^{1-\theta} F(1-\theta, 2-\theta, x)-\lim _{x_{c} \rightarrow 0^{+}}\left(x^{1-\theta}\right)\right] .
\end{aligned}
$$

Hence, if $\theta<1$ we get

$$
\begin{aligned}
T_{0}(x)= & -\frac{1}{\theta} \int_{0}^{x} F(1,1+\theta, z) d z \\
& -\Gamma(\theta-1) x^{1-\theta} F(1-\theta, 2-\theta, x),
\end{aligned}
$$

and taking into account Eq. (50) we see that in this case the expression for the MFPT to the origin is given by Eq. (49) with $x_{c}=0$ :

$$
T_{0}(x)=\int_{0}^{x} U(1,1+\theta, z) d z \quad(\theta<1)
$$


However, we see from Eq. (51) that when $\theta>1, x_{c}^{1-\theta} \rightarrow \infty$ as $x_{c} \rightarrow 0^{+}$and the process takes an infinite average time to reach the origin,

$$
T_{0}(x)=\infty \quad(\theta>1),
$$

which confirms that when $\theta>1$ the singular boundary $x=0$ is unattainable.

\section{Initial value below threshold $\left(x \leqslant x_{c}\right)$}

Now [see Eq. (23)]

$$
\hat{W}_{c}(s \mid x)=\frac{F(s, \theta, x)}{s F\left(s, \theta, x_{c}\right)} .
$$

Hence,

$$
T_{c}(x)=\lim _{s \rightarrow 0}\left[\frac{1}{s} \frac{F\left(s, \theta, x_{c}\right)-F(s, \theta, x)}{F\left(s, \theta, x_{c}\right)}\right] .
$$

In Appendix B we show that the expansion of powers of $s$ of the Kummer function $F(s, \theta, x)$ is

$$
F(s, \theta, x)=1+s F_{1}(x)+O\left(s^{2}\right),
$$

where

$$
F_{1}(x) \equiv \frac{1}{\theta} \int_{0}^{x} F(1,1+\theta, z) d z .
$$

Substituting Eqs. (55) and (56) into Eq. (54) yields

$$
T_{c}(x)=\frac{1}{\theta} \int_{x}^{x_{c}} F(1,1+\theta, z) d z \quad\left(x \leqslant x_{c}\right),
$$

a result we obtained a few years ago [32] in another context and using a different approach. This result that applies for $x \leqslant x_{c}$ jointly with the previous Eq. (49) that applies for $x \geqslant x_{c}$ are presented in Fig. 3. We show there the marked asymmetric behavior of the MFPT depending on whether the initial value $x$ is larger or smaller than the critical value $x_{c}$.

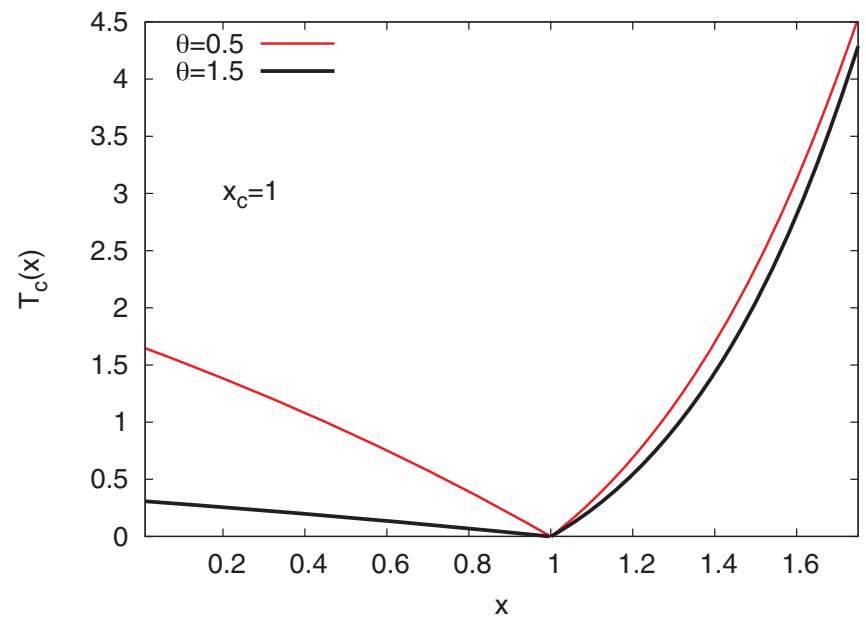

FIG. 3. (Color online) The mean first-passage time as function of the initial value $x$ for two different values of $\theta$ when the critical value $x_{c}$ equals to one. The figure jointly shows all MFPT (57) when $x<x_{c}$ and the MFPT (49) when $x>x_{c}$. Putting together the two mathematical expressions allows us to clearly observe the distinct and asymmetric behavior of the mean first passage depending on whether $x<x_{c}$ or $x>x_{c}$.

\section{The mean escape time}

We close this section by obtaining the time taken by the process $X(t)$ starting at $X(0)=x$ to first leave a given interval $(a, b)$, where $a<x<b$. This is called the escape (or exit) time out of an interval, $\tau_{a b}(x)$, and is formally defined as

$$
\tau_{a b}(x)=\inf \{t \mid a \geqslant X(t) \geqslant b ; a<x<b\} .
$$

The exit time is a random variable characterized by the distribution function

$\operatorname{Prob}\left\{\tau_{a b}(x)<t \mid X(0)=x\right\}=\operatorname{Prob}\{a \geqslant X(t) \geqslant b \mid X(0)=x\}$,

which is precisely the escape probability $W_{a b}(t \mid x)$ discussed in Sec. III. The moments of the exit time are thus defined by

$$
T_{a b}^{(n)}(x)=\int_{0}^{\infty} t^{n} d W_{a b}(t \mid x)
$$

( $n=1,2,3, \ldots)$ and the mean escape time (MET) is the first moment:

$$
T_{a b}^{(1)}(x) \equiv T_{a b}(x)
$$

Proceeding as in Sec. IV A we easily see that the Laplace transform of the escape probability can be written as [see Eq. (41)]

$$
\hat{W}_{a b}(s \mid x)=\sum_{n=0}^{\infty} \frac{(-1)^{n}}{n !} s^{n-1} T_{a b}^{(n)}(x),
$$

from which it follows that [see Eq. (45)]

$$
T_{a b}(x)=\lim _{s \rightarrow 0}\left[\frac{1}{s}-\hat{W}_{a b}(s \mid x)\right] .
$$

Moreover, similarly to the first-passage problem discussed above, we can easily prove that the long-time behavior of the escape probability is also solely determined by the MET [see Eq. (44)]:

$$
W_{a b}(t \mid x) \simeq 1-e^{-t / T_{a b}(x)} \quad(t \rightarrow \infty) .
$$

Plugging the expression for $\hat{W}_{a b}(s \mid x)$ given by Eq. (37) into Eq. (58) and taking into account the small $s$ development of the Kummer functions $U$ and $F$, as expressed respectively by Eqs. (47) and (48) and Eqs. (55) and (56) we obtain after lengthy but otherwise straightforward algebra the following expression of the MET:

$$
T_{a b}(x)=\frac{N_{a b}(x)}{D_{a b}(x)},
$$

where

$$
\begin{aligned}
N_{a b}(x)= & \int_{a}^{x} U(1,1+\theta, z) d z \int_{0}^{b} F(1,1+\theta, z) d z \\
& +\int_{x}^{b} U(1,1+\theta, z) d z \int_{0}^{a} F(1,1+\theta, z) d z \\
& -\int_{a}^{b} U(1,1+\theta, z) d z \int_{0}^{x} F(1,1+\theta, z) d z
\end{aligned}
$$

and

$$
D_{a b}(x)=\int_{a}^{b}[F(1,1+\theta, z)+\theta U(1,1+\theta, z)] d z .
$$




\section{SUMMARY AND CONCLUSIONS}

We have fully addressed the first-passage and escape problems for the Feller process. Let us now summarize the main results obtained. The process is a one-dimensional diffusion defined by a linear drift and a linear diffusion coefficient vanishing at the origin. The Feller process has the property of being positive, a salient characteristic which has earned the process some popularity in modeling several phenomena, from neural activity to financial markets.

Perhaps the best way to define the process is by means of a stochastic differential equation. In the dimensionless units defined in Eq. (2) of Sec. II this reads

$$
d X(t)=-[X(t)-\theta] d t+\sqrt{2 X(t)} d W(t)
$$

(we have dropped the prime in the time variable) where $W(t)$ is the Wiener process and $\theta>0$ is the saturation or normal level to which $X(t)$ is attracted as $t$ increases. The origin is a singular boundary because the noise term vanishes there. In Sec. II we have reviewed the general properties of the processes which were mostly obtained by Feller many years ago. One of these properties refers to the attainability of the origin in which the normal level plays a crucial role. Thus if $\theta \leqslant 1$ the origin is an accessible boundary while if $\theta>1$ it is not.

The bulk of the paper is developed in Secs. III and IV where the first-passage and escape properties of the Feller process are thoroughly analyzed. The first-passage problem refers to the crossing by the process of a certain preassigned critical level or threshold $x_{c}$ while the escape problem refers to the departure of some interval $(a, b)$.

The first-passage properties are fully characterized by the hitting, or first-passage, probability, defined as the probability of first reaching the threshold $x_{c}$ at time $t$ or before. We denote by $W_{c}(t \mid x)$ this probability, where $x$ is the initial value. This probability depends on whether the process is initially below $\left(x \leqslant x_{c}\right)$ or above $\left(x \geqslant x_{c}\right)$ the threshold. We have obtained exact expressions for the Laplace transform of the hitting probability,

$$
\hat{W}_{c}(s \mid x)=\int_{0}^{\infty} e^{-s t} W_{c}(t \mid x) d t,
$$

which are summarized as

$$
\hat{W}_{c}(s \mid x)= \begin{cases}\frac{F(s, \theta, x)}{s F\left(s, \theta, x_{c}\right)}, & x \leqslant x_{c}, \\ \frac{U(s, \theta, x)}{s U\left(s, \theta, x_{c}\right)}, & x \geqslant x_{c},\end{cases}
$$

where $F$ and $U$ are Kummer functions.
In general these expressions for the Laplace transform of the hitting probability cannot be inverted exactly in an analytical fashion and one has to resort to the numerical inversion. There are some instances, however, in which we have been able to obtain analytical expressions in real time. This is the case of hitting the origin which has a significant interest in the firing of neurons and also in the Heston volatility model of the financial analysis. We denote by $W_{0}(t \mid x)$ the first-passage probability to threshold $x_{c}=0$; we have shown that

$$
W_{0}(t \mid x)= \begin{cases}\frac{1}{\Gamma(1-\theta)} \Gamma\left(1-\theta, \frac{x e^{-t}}{1-e^{-t}}\right), & \theta<1, \\ 0, & \theta>1,\end{cases}
$$

where $\Gamma(a, z)$ is the incomplete Gamma function. If $\theta<1$, $W_{0}(t \mid x) \rightarrow 1$ as $t \rightarrow \infty$. This is the expected behavior since when $\theta<1$ crossing the origin is a sure event as time grows. The way $W_{0}$ approaches unity is explicitly given by the following combination of a power law in the initial position and an exponential time decay:

$$
\begin{aligned}
& W_{0}(t \mid x) \\
& \quad=1-\frac{1}{\Gamma(2-\theta)} x^{1-\theta} e^{-(1-\theta) t}+O\left(e^{-(2-\theta) t}\right) \quad(\theta<1) .
\end{aligned}
$$

Another instance in which we have been able to obtain an (approximate) expression for the first-passage probability in real time is when threshold is large. In such a case

$W_{c}(t \mid x) \simeq \frac{1}{\Gamma(\theta)} x_{c}^{\theta} e^{-x_{c}} \sum_{n=0}^{\infty} \frac{1}{(\theta)_{n}} \frac{x^{n}}{n !} \Gamma\left(n, x_{c} e^{-t}\right) \quad\left(x_{c} \rightarrow \infty\right)$,

where $\Gamma\left(n, x_{c} e^{-t}\right)$ is the incomplete Gamma function.

The escape problem is completely characterized by the escape probability, $W_{a b}(t \mid x)$, defined as the probability of first leaving a given interval $(a, b)$. It is complementary to the survival probability $S_{a b}$ :

$$
W_{a b}(t \mid x)=1-S_{a b}(t \mid x),
$$

where $S_{a b}(t \mid x)$ is the probability that the process has not exited $(a, b)$ at time $t$ or during any previous instant of time. Formally,

$$
\begin{aligned}
& S_{a b}(t \mid x) \\
& \quad=\operatorname{Prob}\left\{X\left(t^{\prime}\right) \in(a, b), 0 \leqslant t^{\prime} \leqslant t \mid X(0)=x \in(a, b)\right\} .
\end{aligned}
$$

For the Feller process we have been able to obtain the exact expression for the Laplace transform of the escape probability which turns out to be more involved than the first-passage probability. It reads

$$
\hat{W}_{a b}(s \mid x)=\frac{[U(s, \theta, b)-U(s, \theta, a)] F(s, \theta, x)-[F(s, \theta, b)-F(s, \theta, a)] U(s, \theta, x)}{s[F(s, \theta, a) U(s, \theta, b)-F(s, \theta, b) U(s, \theta, a)]},
$$

where $a \leqslant x \leqslant b$, and $F$ and $U$ are Kummer functions.

We have next addressed the problem of the mean firstpassage time (MFPT) and the mean exit time (MET). We have shown that in terms of the first-passage time moments $T_{n}\left(x \mid x_{c}\right)(n=1,2,3, \ldots)$ - of which the MFPT corresponds to $n=1, T_{1}\left(x \mid x_{c}\right) \equiv T_{c}(x)$ - the Laplace transform of the hitting probability reads

$$
\hat{W}_{c}(s \mid x)=\frac{1}{s}+\sum_{n=1}^{\infty} \frac{(-1)^{n}}{n !} s^{n-1} T_{n}\left(x \mid x_{c}\right) .
$$


The MFPT to the threshold $x_{c}$ is then given by

$$
T_{c}(x)=\lim _{s \rightarrow 0}\left[\frac{1}{s}-\hat{W}_{c}(s \mid x)\right] .
$$

From these expressions we have been able to obtain, in terms of the MFPT, the following long-time asymptotic expression of the hitting probability:

$$
W_{c}(t \mid x) \simeq 1-e^{-t / T_{c}(x)} \quad(t \rightarrow \infty) .
$$

For the Feller process this analysis has led to different results according to whether initially the system is placed below or above the threshold:

$$
T_{c}(x)= \begin{cases}(1 / \theta) \int_{x_{c}}^{x} F(1,1+\theta, z) d z, & x \leqslant x_{c}, \\ \int_{x_{c}}^{x} U(1,1+\theta, z) d z, & x \geqslant x_{c} .\end{cases}
$$

The MFPT to reach the origin, $T_{0}(x)$, has also been analyzed with the result

$$
T_{0}(x)= \begin{cases}\int_{0}^{x} U(1,1+\theta, z) d z, & \theta<1, \\ \infty, & \theta>1,\end{cases}
$$

which constitutes an additional proof of the fact that when $\theta>1$ the singular boundary $x=0$ is unattainable.

The analysis of the MFPT can be exactly carried out for the MET. The resulting expressions relating the MET with the escape probability are formally the same as those relating the MFPT with the hitting probability as can be seen in Sec. IV C. Thus, for instance,

$$
T_{a b}(x)=\lim _{s \rightarrow 0}\left[\frac{1}{s}-\hat{W}_{a b}(s \mid x)\right]
$$

and

$$
W_{a b}(t \mid x) \simeq 1-e^{-t / T_{a b}(x)} \quad(t \rightarrow \infty)
$$

where $W_{a b}(t \mid x)$ and $T_{a b}(x)$ are the escape probability and the MET, respectively. In the case of the Feller process the explicit expression for the MET is given in Eqs. (60)-(62).

Let us finally mention that the extension of the above results to the study of the extreme values attained by the process, such as the maximum and minimum values, as well as their application to financial time series - in particular the volatility, is under present research and we expect getting a number of results very soon.

\section{ACKNOWLEDGMENTS}

Partial financial support from the Ministerio de Ciencia e Innovación under Contract No. FIS 2009-09689 is acknowledged.

\section{APPENDIX A: THE PROBABILITY DENSITY FUNCTION}

The solution to the problem (5) and (6) is more conveniently addressed by its Laplace transform with respect to $x$ :

$$
\hat{p}\left(\sigma, t \mid x_{0}\right)=\int_{0}^{\infty} e^{-\sigma x} p\left(x, t \mid x_{0}\right) d x .
$$

Taking into account condition (7), the transformed problem (5) and (6) reads

$$
\begin{gathered}
\frac{\partial \hat{p}}{\partial t}+\sigma(1+\sigma) \frac{\partial \hat{p}}{\partial \sigma}=-\theta \sigma \hat{p}, \\
\hat{p}\left(\sigma, 0 \mid x_{0}\right)=e^{-\sigma x_{0}}
\end{gathered}
$$

Equation (A2) is a linear partial differential equation of first order whose solution can be obtained by the method of characteristics [33]. In effect, let the function $h(\sigma)$ be defined by the characteristic of Eq. (A2), $h^{\prime}(\sigma)=-[\sigma(1+\sigma)]^{-1}$; that is,

$$
h(\sigma)=\ln \left(\frac{1+\sigma}{\sigma}\right) .
$$

Then the solution of Eq. (A2), as can be rightly seen by direct substitution, is [33]

$$
\hat{p}\left(\sigma, t \mid x_{0}\right)=(1+\sigma)^{-\theta} \psi(t+h(\sigma)),
$$

where $\psi(z)$ is an arbitrary function to be determined by the initial condition (A3), i.e.,

$$
\psi(h(\sigma))=(1+\sigma)^{\theta} e^{-\sigma x_{0}},
$$

which implies, after inverting Eq. (A4) to write $\sigma$ in terms of $h$, that

$$
\psi(z)=\left(1-e^{-z}\right)^{-\theta} \exp \left\{-\frac{x_{0} e^{-z}}{1-e^{-z}}\right\} .
$$

Substituting this into Eq. (A5) we finally obtain

$$
\hat{p}\left(\sigma, t \mid x_{0}\right)=\frac{1}{\left[1+\sigma\left(1-e^{-t}\right)\right]^{\theta}} \exp \left\{-\frac{\sigma x_{0} e^{-t}}{1+\sigma\left(1-e^{-t}\right)}\right\} .
$$

Let us now proceed to the Laplace inversion of Eq. (A6). Calling

$$
a=1-e^{-t}, \quad b=x_{0} e^{-t},
$$

simple algebraic manipulations followed by a power expansion yield

$$
\begin{aligned}
\exp \left\{-\frac{b \sigma}{1+a \sigma}\right\} & =e^{-b / a} \exp \left\{-\frac{b}{a(1+a \sigma)}\right\} \\
& =e^{-b / a} \sum_{n=0}^{\infty} \frac{(b / a)^{n}}{n !(1+a \sigma)^{n}} .
\end{aligned}
$$

Plugging into Eq. (A6) we get

$$
\hat{p}\left(\sigma, t \mid x_{0}\right)=e^{-b / a} \sum_{n=0}^{\infty} \frac{(b / a)^{n}}{n !(1+a \sigma)^{n+\theta}} .
$$

Let us denote by $\mathcal{L}^{-1}\{\hat{f}(\sigma)\}=f(x)$ the operation of Laplace inverting $\hat{f}(\sigma)$ and recall the standard property

$$
\mathcal{L}^{-1}\{\hat{f}(a \sigma+1)\}=\frac{1}{a} e^{-x / a} f(x / a)
$$

and also

$$
\mathcal{L}^{-1}\left\{\frac{1}{\sigma^{n+\theta}}\right\}=\frac{x^{n+\theta-1}}{\Gamma(n+\theta)} .
$$

Then the Laplace inversion of Eq. (A8) yields

$$
p\left(x, t \mid x_{0}\right)=\frac{1}{a} e^{-(x+b) / a} \sum_{n=0}^{\infty} \frac{(b / a)^{n}(x / a)^{n+\theta-1}}{n ! \Gamma(n+\theta)},
$$


which after simple manipulations reads

$$
p\left(x, t \mid x_{0}\right)=\frac{1}{a}\left(\sqrt{\frac{x}{b}}\right)^{\theta-1} e^{-(x+b) / a} \sum_{n=0}^{\infty} \frac{(\sqrt{b x} / a)^{2 n+\theta-1}}{n ! \Gamma(n+\theta)} .
$$

We recognize the series appearing in this equation as the expression of a modified Bessel function. Indeed

$$
I_{v}(z)=\sum_{n=0}^{\infty} \frac{(z / 2)^{2 n+v}}{n ! \Gamma(n+v+1)}
$$

is the modified Bessel function of order $v$ [28]. Therefore,

$$
p\left(x, t \mid x_{0}\right)=\frac{1}{a}\left(\frac{x}{b}\right)^{(\theta-1) / 2} e^{-(x+b) / a} I_{\theta-1}\left(\frac{2 \sqrt{b x}}{a}\right)
$$

and, reverting to the original notation [see Eq. (A7)], we write

$$
\begin{aligned}
p\left(x, t \mid x_{0}\right)= & \frac{1}{1-e^{-t}}\left(\frac{x e^{-t}}{x_{0}}\right)^{(\theta-1) / 2} \\
& \times \exp \left\{-\frac{x+x_{0} e^{-t}}{1-e^{-t}}\right\} I_{\theta-1}\left(\frac{2 \sqrt{x x_{0} e^{-t}}}{1-e^{-t}}\right),
\end{aligned}
$$

which is Eq. (8).

\section{APPENDIX B: EXPANSIONS FOR $F(s, \theta, x)$ AND $U(s, \theta, x)$}

In terms of the Pochhammer's symbol $(a)_{n}=\Gamma(a+$ $n) / \Gamma(a)$, Kummer function $F$ is defined as the series [28]

$$
F(s, \theta, x)=\sum_{n=0}^{\infty} \frac{(s)_{n}}{(\theta)_{n}} \frac{x^{n}}{n !} .
$$

Since $(s)_{0}=1$ and

$(s)_{n}=s(s+1)(s+2) \cdots(s+n-1)=s(n-1) !+O\left(s^{2}\right)$,

we have

$$
F(s, \theta, x)=1+s \sum_{n=1}^{\infty} \frac{1}{(\theta)_{n}} \frac{x^{n}}{n}+O\left(s^{2}\right) .
$$

In the sum of the right-hand side we make the replacement $n \rightarrow n+1$ and take into account that $(\theta)_{n+1}=\theta(\theta+1)_{n}$; we thus write

$$
\begin{aligned}
\sum_{n=1}^{\infty} \frac{1}{(\theta)_{n}} \frac{x^{n}}{n} & =\frac{1}{\theta} \sum_{n=0}^{\infty} \frac{1}{(\theta+1)_{n}} \frac{x^{n+1}}{n+1} \\
& =\frac{1}{\theta} \sum_{n=0}^{\infty} \frac{1}{(\theta+1)_{n}} \int_{0}^{x} z^{n} d z .
\end{aligned}
$$

We can easily see that $(1)_{n}=n$ !, hence

$$
\sum_{n=1}^{\infty} \frac{1}{(\theta)_{n}} \frac{x^{n}}{n}=\frac{1}{\theta} \int_{0}^{x}\left[\sum_{n=0}^{\infty} \frac{(1)_{n}}{(\theta+1)_{n}} \frac{z^{n}}{n !}\right] d z
$$

which, after recalling the definition of the confluent hypergeometric function $F$, Eq. (20), yields

$$
\sum_{n=1}^{\infty} \frac{1}{(\theta)_{n}} \frac{x^{n}}{n}=\frac{1}{\theta} \int_{0}^{x} F(1,1+\theta, z) d z
$$

Substituting into Eq. (B1) we get

$$
F(s, \theta, x)=1+s F_{1}(x)+O\left(s^{2}\right),
$$

where

$$
F_{1}(x) \equiv \frac{1}{\theta} \int_{0}^{x} F(1,1+\theta, z) d z
$$

The small $s$ expansion of the Kummer function of the second kind $U(s, \theta, x)$ is a bit more involved. We start from the definition of $U$ in terms of $F$ [see Eq. (21)],

$$
\begin{aligned}
U(s, \theta, x)= & \frac{\Gamma(1-\theta)}{\Gamma(1-\theta+s)} F(s, \theta, x) \\
& +\frac{\Gamma(\theta-1)}{\Gamma(s)} x^{\theta-1} F(1-\theta+s, 2-\theta, x),
\end{aligned}
$$

then expand

$$
\begin{aligned}
\Gamma(1-\theta+s) & =\Gamma(1-\theta)+s \Gamma^{\prime}(1-\theta)+O\left(s^{2}\right) \\
& =\Gamma(1-\theta)\left[1+s \psi(1-\theta)+O\left(s^{2}\right)\right]
\end{aligned}
$$

where $\psi(z)=\Gamma^{\prime}(z) / \Gamma(z)$ is the psi function [28]. Also [34]

$$
\Gamma(s)=\frac{1}{s}\left[1-\gamma s+O\left(s^{2}\right)\right],
$$

where $\gamma=0.5772 \cdots$ is Euler's constant. Plugging Eqs. (B2), (B5), and (B6) into Eq. (B4) we get

$$
U(s, \theta, x)=1+s U_{1}(x)+O\left(s^{2}\right),
$$

where

$$
U_{1}(x) \equiv F_{1}(x)-\psi(1-\theta)+\Gamma(\theta-1) F(1-\theta, 2-\theta, x) .
$$

Let us finally show that a more convenient form for $U_{1}(x)$ is given by

$$
U_{1}(x)=-\psi(1-\theta)-\int^{x} U(1,1+\theta, z) d z
$$

In effect, recalling the definition of $F_{1}(x)$ given in Eq. (B3) and using the integration rule $\int x^{c-1} F(a, c, x) d x=$ $\left(x^{c} / c\right) F(a, c+1, x)$, we have

$$
\begin{aligned}
U_{1}(x)= & -\psi(1-\theta)-\int\left[\frac{\Gamma(-\theta)}{\Gamma(1-\theta)} F(1,1+\theta, x)\right. \\
& \left.+\Gamma(\theta) x^{\theta} F(1-\theta, 1-\theta, x)\right] d x,
\end{aligned}
$$


where we have used the well-known property $\Gamma(1+z)=z \Gamma(z)$ to write $1 / \theta=-\Gamma(-\theta) / \Gamma(1-\theta)$ and $(1-\theta) \Gamma(\theta-1)=$ $-\Gamma(\theta)$. Note that the integrand is precisely the Kummer function of the second kind

$$
U(1,1+\theta, x)=\frac{\Gamma(-\theta)}{\Gamma(1-\theta)} F(1,1+\theta, x)+\Gamma(\theta) x^{\theta} F(1-\theta, 1-\theta, x)
$$

[see Eq. (B4) with $s=1$ and $\theta$ replaced by $1+\theta$ ]. We have thus proven Eq. (B9).

[1] W. Feller, Ann. Math. 54, 173 (1951).

[2] W. Feller, Ann. Math. 55, 468 (1952).

[3] W. Feller, Trans. Am. Math. Soc. 71, 1 (1954).

[4] C. W. Gardiner, Handbook of Stochastic Methods (SpringerVerlag, Berlin, 1985).

[5] L. M. Ricciardi, Diffusion Processes and Related Topics in Biology (Springer-Verlag, Berlin, 1977).

[6] R. M. Capocelli and L. M. Ricciardi, J. Theor. Biol. 40, 369 (1973).

[7] W. Gerstner and W. M. Kistler, Spiking Neuron Models (Cambridge University Press, Cambridge, 2002).

[8] V. Lanska, P. Lansky, and C. E. Smith, J. Theor. Biol. 166, 393 (1994).

[9] P. Lansky, L. Sacerdote, and F. Tomassetti, Biol. Cybern. 73, 457 (1995).

[10] S. Ditlevsen, Phys. Rev. E 73, 061910 (2006).

[11] P. Lansky and S. Ditlevsen, Biol. Cybern. 99, 253 (2008).

[12] E. Bibbona, P. Lansky, and R. Sirovich, Phys. Rev. E 81, 031916 (2010).

[13] R. Jolivet, R. Kobayashi, A. Rauch, R. Naud, S. Shinomoto, and W. Gerstner, J. Neurosci. Methods 169, 417 (2008).

[14] T. Tchumatchenko, A. Malyshev, T. Geisel, M. Volgushev, and F. Wolf, Phys. Rev. Lett. 104, 058102 (2010).

[15] R. M. Capocelli and L. M. Ricciardi, Theoretical Population Biology 5, 28 (1974).

[16] J. D. Murray, Mathematical Biology (Springer, Berlin, 2002).

[17] S. Azaele, A. Maritan, E. Bertuzzo, I. Rodriguez-Iturbe, and A. Rinaldo, Phys. Rev. E 81, 051901 (2010).

[18] S. Azaele, S. Pigolotti, J. R. Banavar, and A. Maritan, Nature (London) 444, 926 (2006).
[19] J. C. Cox, J. E. Ingersoll, and S. A. Ross, Econometrica 53, 385 (1985).

[20] J. C. Hull, Options, Futures, and Other Derivatives (Prentice Hall, London, 2011).

[21] S. L. Heston, Rev. Financ. Stud. 6, 327 (1993).

[22] A. A. Dragulescu and V. M. Yakovenko, Quant. Fin. 2, 443 (2002).

[23] S. Redner, A Guide to First-Passage Processes (Cambridge University Press, Cambridge, 2001).

[24] T. Verechtchaguina, I. M. Sokolov, and L. Schimansky-Geier, Phys. Rev. E 73, 031108 (2006).

[25] S. Condamin, O. Bénichou, V. Tejedor, R. Voituriez, and J. Klafter, Nature (London) 450, 77 (2007).

[26] M. F. Shlesinger, Nature (London) 450, 40 (2007).

[27] G. H. Weiss, First Passage Time Problems in Chemical Physics, in Advances in Chemical Physics, Vol. 13, edited by I. Prigogine (J. Wiley, Hoboken, NJ, 2007).

[28] W. Magnus, F. Oberhettinger, and R. P. Soni, Formulas and Theorems for the Special Functions of Mathematical Physics (Springer-Verlag, Berlin, 1966).

[29] H. Stehfest, Communications of the Association for Computing Machinery 13, 47 (1970).

[30] G. E. Roberts and H. Kaufman, Table of Laplace Transforms (W. B. Sauders, Philadelphia, 1966).

[31] R. A. Handelsman and J. S. Lew, SIAM J. Math. Anal. 5, 425 (1974).

[32] J. Masoliver and J. Perelló, Phys. Rev. E 75, 046110 (2007).

[33] R. Courant and D. Hilbert, Methods of Mathematical Physics, Vol. 2 (J. Wiley-VCH, New York, 1989).

[34] M. Abramowitz and I. A. Stegun, editors, Hanbook of Mathematical Functions (Dover, New York, 1972). 